\title{
Concepts of barodesy
}

\author{
Gertraud Medicus, Wolfgang Fellin, Dimitrios Kolymbas and Fabian Schranz
}

This is the post-print of Concepts of Barodesy, published online 10 August 2019. https: //doi.org/10.1007/978-3-030-14987-1_12

It should be cited as: Medicus, G., Fellin, W., Kolymbas, D. and Schranz, F. (2019) Concepts of Barodesy. In: Wu W. (eds) Desiderata Geotechnica. Springer Series in Geomechanics and Geoengineering. Springer, Cham. doi: 10.1007/978-3-030-14987-1_12

\begin{abstract}
Barodesy is a frame for constitutive modeling of soils based on their asymptotic properties. This frame allows to derive the constitutive relation by reasoning on general properties of granular materials. The so obtained constitutive relation is a single tensorial equation that expresses the evolution of stress in dependence of the deformation. Common concepts of soil mechanics, such as critical states, barotropy (i.e. the dependence of stiffness and strength on the stress level), pyknotropy (i.e. the dependence of stiffness and strength on density) and a stress-dilatancy relation are comprised in the presented model.
\end{abstract}

\section{Introduction}

Compared with elastoplasticity, barodesy is an alternative frame of constitutive models for soils. The structure of the theory and a simple calibration procedure are outlined in this article, and some results of simulations of element tests are also presented. Barodesy holds, with small adjustments, for both types of soil, sand and clay. Clay, being also a particulate material consisting of minute particles has a behavior very similar to sand. Both exhibit critical states. In addition, an asymptotic behavior similar to the one described by Goldscheider for sand (see Sect.4) was observed by Topolnicki in experiments with Kaolin clay in a Hambly type biaxial apparatus [25]. However, there are some differences that mainly arise from the fact that the stiffness of sand in monotonic compression is much higher than that of normally consolidated clay. In other words, sand has the tendency to jam at monotonic compression.

Gertraud Medicus · Wolfgang Fellin · Dimitrios Kolymbas · Fabian Schranz Geotechnik und Tunnelbau, Universität Innsbruck, Austria

e-mail: Gertraud.Medicus@uibk.ac.at 


\section{On the name 'barodesy'}

Usually the word 'plasticity' is used with prefixes such as hypo-, para-, hyper- etc. to denote the various constitutive models. For granular materials, however, the words 'elasticity' and 'plasticity' (to the extend the latter is associated with notions such as yield surface, elastic regime etc., originally created for metals) can be avoided. Yield surfaces and the other concepts of plasticity theory may prejudice our perception and sometimes obscure soil mechanics, which suffers from a long lasting fragmentation in constitutive modelling [12]. The name 'barodesy' has therefore been coined by Kolymbas motivated by the fact that granular materials gain their stiffness $(\delta \varepsilon \sigma \iota \varsigma=$ bond, hence stiffening, hardening) from externally applied pressure ( $\beta \alpha \alpha \rho \varsigma)$. Thus, the names 'barodesy' and 'barodetic' are proposed for granular materials to distinguish them from what traditionally is denoted as 'elastic' or 'plastic'.

\subsection{Barodesy and hypoplasticity}

As in hypoplasticity [10, 11, 2, $1,7,21,20,9,27,29,28,15]$, the barodetic constitutive equation has the general form

$$
\stackrel{\circ}{\boldsymbol{T}}=\boldsymbol{h}(\boldsymbol{T}, \boldsymbol{D}, e)
$$

to describe the co-rotational stress rate $\stackrel{\circ}{\boldsymbol{T}}$ as a function of stress $\boldsymbol{T}$, stretching $\boldsymbol{D}$ and void ratio $e$. However, barodesy has a different design than hypoplasticity. In the original versions of hypoplasticity published by Kolymbas, trial and error was applied by general principles of objectivity and representation theorems for tensor-valued functions. In barodesy, the amount of trial and error has been reduced in favour of reasoning on asymptotic behaviour of granulates. Asymptotic states matter not only from conceptual reasons but also from the experimental viewpoint: With long monotonic deformations, initial disturbances, related e.g. with sample preparation, fade out and do no more influence the results.

\section{Symbols and notation}

As usual in mechanics, the symbols $\sigma$ and $\boldsymbol{T}$ denotes the stress, and $\varepsilon$ denotes the strain. Tensors are usually denoted either in the index notation or in the symbolic notation with boldface letters. Compared to the index notation, the symbolic notation facilitates insight into the prevailing relationships. Therefore, in this paper the symbolic notation, as introduced in [26], is mainly followed.

\section{Empirical basis of barodesy}

Of basic importance for the following is the notion of a proportional path. Proportional stress and strain paths are characterized by constant ratios of the principal values $\sigma_{1}: \sigma_{2}: \sigma_{3}$ and $\varepsilon_{1}: \varepsilon_{2}: \varepsilon_{3}$, respectively.

There are two basic experimental findings for sand:

1. Starting from the stress-free state, proportional strain paths lead to proportional stress paths. 
Table 1 Symbols and notation

\begin{tabular}{|c|c|}
\hline Symbol & Description \\
\hline$T$ & $\begin{array}{l}\text { Cauchy-stress. Its components are } \sigma_{i j} \text {. Its principal components are denoted as } \\
\sigma_{1}, \sigma_{2}, \sigma_{3} \text {. }\end{array}$ \\
\hline$D$ & $\begin{array}{l}\text { stretching tensor, i.e. the symmetric part of the velocity gradient } \nabla v \text {. It can be set } \\
\text { approximately equal to the strain rate, } D_{i j} \approx \dot{\varepsilon}_{i j} \text {. }\end{array}$ \\
\hline $\operatorname{tr} D$ & $\begin{array}{l}\text { trace of } \boldsymbol{D} \text { is the scalar quantity } \operatorname{tr} \boldsymbol{D}=D_{11}+D_{22}+D_{22} \text {, which denotes the rate of } \\
\text { volume change: } \operatorname{tr} \boldsymbol{D}=\dot{\varepsilon}_{\mathrm{v}}=\dot{\varepsilon}_{11}+\dot{\varepsilon}_{22}+\dot{\varepsilon}_{33} .\end{array}$ \\
\hline$e$ & $\begin{array}{l}\text { void ratio, i.e. the ratio } V_{p} / V_{s} \text {, where } V_{p} \text { and } V_{s} \text { are the volumes of pores and solids } \\
\text { (grains), respectively. }\end{array}$ \\
\hline$\| \boldsymbol{A} \mid$ & the value of a tensor is its Euclidean norm: $\|A\|=\sqrt{\operatorname{tr} A^{2}}$. \\
\hline $\boldsymbol{A}^{0}$ & normalized tensor, i.e. $\boldsymbol{A}^{0}=\boldsymbol{A} /\|\boldsymbol{A}\|$. Clearly, $\boldsymbol{A}^{0}$ is a unit tensor with $\left\|\boldsymbol{A}^{0}\right\|=1$. \\
\hline$\delta=\operatorname{tr} D^{0}$ & This quantity is a measure of dilatancy. \\
\hline$\stackrel{\circ}{T}$ & objective stress $r$ \\
\hline$c_{i}$ & $\begin{array}{l}\text { constants. It facilitates reading to clearly denote the constants in mathematical } \\
\text { functions as such and to distinguish them from quantities that depend on other } \\
\text { variables. }\end{array}$ \\
\hline
\end{tabular}

2. Starting from a non-vanishing stress state and applying a proportional strain path leads asymptotically to the proportional stress path that would be obtained starting from the stress-free state.

These two rules are inferred by GOLDSCHEIDER from his test results obtained with rectilinear extensions of sand [6] (cf. also [16]). These tests have been carried out in a so-called true triaxial apparatus, which allows to apply rectilinear extensions (i.e. motions without rotation of the principal axes of deformation) independently in all three directions of space.

\section{Virgin proportional paths}

We first consider proportional strain paths starting form the stress-free state. Such paths can be volume-decreasing (we will call them 'consolidations'), characterized by $\operatorname{tr} \boldsymbol{D}<0$, or volume preserving ('isochoric' or 'undrained'), characterized by $\operatorname{tr} \boldsymbol{D}=0$, or volume increasing, characterized by $\operatorname{tr} \boldsymbol{D}>0$. Clearly, the latter are not feasible with cohesionless sand.

Let us denote with $\boldsymbol{R}$ a tensor that has the direction of a proportional stress path. The question arises, how does $\boldsymbol{R}$ depend on the direction of the corresponding proportional strain path? The latter is characterized by the direction of stretching $\boldsymbol{D}$, i.e. by the normalized stretching $\boldsymbol{D}^{0}$. How can we determine the relation $\boldsymbol{R}\left(\boldsymbol{D}^{0}\right)$ ? This question can be easily answered if we observe that all consolidations are mapped into a specific part of the principal stress space, which is formed by the stress components $\sigma_{1}, \sigma_{2}$ and $\sigma_{3}$. This part is the octant, where all principal stresses are compressive, i.e. negative. Hence, the product $\sigma_{1} \sigma_{2} \sigma_{3}$ must also be negative. Now, for a proportional stress path we have $\sigma_{i}=\mu R_{i}(\boldsymbol{D}), i=1,2,3$, where $\mu$ simply denotes the proportionality of $\sigma_{i}$ and $R_{i} \prod$ With $R\left(D_{i}\right):=R_{i}$, the following condition must hold:

$$
R\left(D_{1}\right) R\left(D_{2}\right) R\left(D_{3}\right)<0 \quad \text { for } \quad \operatorname{tr} \boldsymbol{D}=D_{1}+D_{2}+D_{3}<0
$$

\footnotetext{
${ }^{1}$ Herein, $R_{i}(\boldsymbol{D})$ are the principal values of $\boldsymbol{R}(\boldsymbol{D})$.
} 
Fig. 1 Critical stress states in the deviatoric plane $(\operatorname{tr} \boldsymbol{T}=-500 \mathrm{kPa})$ : normally consolidated San Francisco Bay $\operatorname{Mud}\left(\bullet, \varphi_{c}=30.6^{\circ}\right)[14$ is compared with MatsuokaNakai, Mohr-Coulomb and barodesy. Barodesy and Matsuoka-Nakai practically coincide [5].

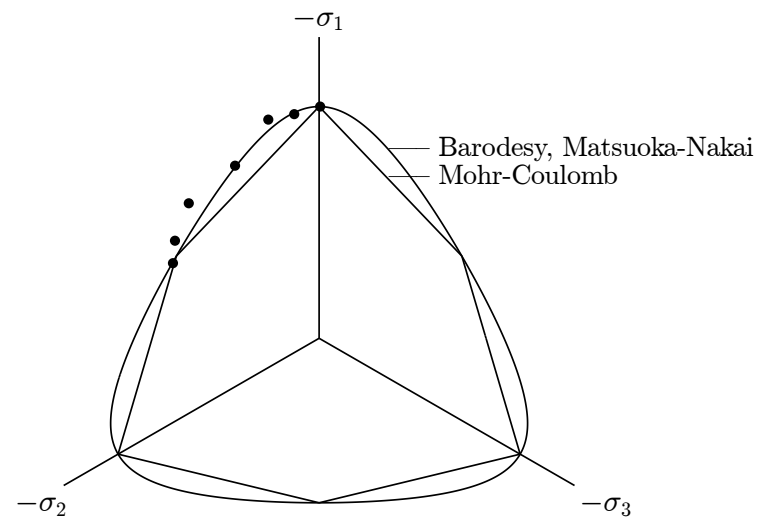

This implies that the product $R\left(D_{1}\right) R\left(D_{2}\right) R\left(D_{3}\right)$ must be a function of the sum $D_{1}+D_{2}+D_{3}$, a requirement which is fulfilled by the exponential mapping

$$
\boldsymbol{R}(\boldsymbol{D})=-\exp \left(a \boldsymbol{D}^{0}\right)
$$

$a$ depends on dilatancy $\delta$, and for $\delta=0$ it obtains the value $a_{0}$. It should be added that Goldscheider's rules have, as every rule, some exceptions in detail. Thus, it is known that stress paths obtained with oedometric deformation are not strictly proportional, i.e. $K_{0}:=\sigma_{2} / \sigma_{1}$ is not strictly constant. Such 'details' can be captured by slight modifications of barodesy [4], but they are omitted here for simplicity.

\subsection{Critical state surface}

Equation 3 maps all volume-reducing $(\operatorname{tr} \boldsymbol{D}<0)$ proportional strain paths into a cone in the stress space with appex at $\boldsymbol{T}=\mathbf{0}$, which can be called the $\boldsymbol{R}$-cone. Its boundary corresponds to paths with $\operatorname{tr} \boldsymbol{D}=0$ and is thus the critical state surface. Consider the intersection of the $\boldsymbol{R}$-cone with a plane $\operatorname{tr} \boldsymbol{T}=$ const, as shown in Figure 1 This curve expresses the critical limit state in a so-called deviatoric plane of the stress space. The mathematical representation of this curve can be easily derived from equation 3 : Eliminating $\boldsymbol{D}^{0}$ from 3 we obtain:

$$
\boldsymbol{D}^{0}=\frac{1}{a} \ln (-\boldsymbol{R}) .
$$

The requirement $\operatorname{tr} D^{0}=0$ results in $\ln \left(-R_{1} R_{2} R_{3}\right)=0$ or $R_{1} R_{2} R_{3}=-1$. From the additional requirement $\left\|\boldsymbol{D}^{0}\right\|=1$ we obtain:

$$
\left[\ln \left(-R_{1}\right)\right]^{2}+\left[\ln \left(-R_{2}\right)\right]^{2}+\left[\ln \left(-R_{3}\right)\right]^{2}=a_{0}^{2} .
$$

For the here considered proportional paths holds: $\boldsymbol{T}=\mu \boldsymbol{R}, 0<\mu<\infty$, hence we can replace in this equation $\boldsymbol{R}$ by $\boldsymbol{T} / \mu$ and obtain finally the equation of critical states in the stress space 2

$$
\left(\ln \frac{\sigma_{1}}{\sqrt[3]{\sigma_{1} \sigma_{2} \sigma_{3}}}\right)^{2}+\left(\ln \frac{\sigma_{2}}{\sqrt[3]{\sigma_{1} \sigma_{2} \sigma_{3}}}\right)^{2}+\left(\ln \frac{\sigma_{3}}{\sqrt[3]{\sigma_{1} \sigma_{2} \sigma_{3}}}\right)^{2}=a_{0}^{2} .
$$

\footnotetext{
${ }^{2}$ From $\sigma_{1} \sigma_{2} \sigma_{3}=-1 / \mu^{3}$ we obtain $\mu=-1 / \sqrt[3]{\sigma_{1} \sigma_{2} \sigma_{3}}$ and replace in equation ${ }_{5} R_{i}$ by $T_{i} / \mu$.
} 
Equation $\sqrt{6}$ is homogeneous of the 0 -th degree in $\boldsymbol{T}$ and describes thus a conical surface in the stress space with apex at $\boldsymbol{T}=\mathbf{0}$. Its intersection with a plane $\operatorname{tr} \boldsymbol{T}=$ const is shown in Figure 1 Its shape practically coincides [5] with the curve obtained by the well-known expression of MATSUOKA \& NAKAI:

$$
\frac{\left(\sigma_{1}+\sigma_{2}+\sigma_{3}\right)\left(\sigma_{1} \sigma_{2}+\sigma_{1} \sigma_{3}+\sigma_{2} \sigma_{3}\right)}{\sigma_{1} \sigma_{2} \sigma_{3}}=\text { const } .
$$

Equation (3) maps not only isochoric but also volume-reducing and volume increasing proportional strain paths to the corresponding proportional stress paths. It proves that this mapping gets more realistic if we let $a$ depend on dilatancy $\delta$, e.g. as

$$
a(\delta)=-30+c_{3} \frac{|\delta-\sqrt{2}|^{c_{2}}}{(1+|\delta-\sqrt{2}|)^{c_{1}}} .
$$

This modification does not affect isochoric (i.e. undrained) proportional strain paths with $\delta=0$. Thus, we replace equation 3 by equation

$$
\boldsymbol{R}(\boldsymbol{D})=-\exp \left[\left(-30+c_{3} \frac{|\delta-\sqrt{2}|^{c_{2}}}{(1+|\delta-\sqrt{2}|)^{c_{1}}}\right) \boldsymbol{D}^{0}\right]
$$

This equation is capable to map arbitrary proportional paths (cf. Fig. 2).

For the calibration of $c_{1}, c_{2}$ and $c_{3}$ the stress ratios $K=\sigma_{1} / \sigma_{2}$ from the oedometer test (e.g. Jáky's law $K_{0}=1-\sin \varphi_{\mathrm{c}}$ ), the critical state $\left(K_{\mathrm{c}}=\frac{1-\sin \varphi_{\mathrm{c}}}{1+\sin \varphi_{\mathrm{c}}}\right)$ and a dilatant stress state (e.g. according to Chu and Lo [3]) can be used (marked with circles in Fig. 2]. All these stress states can be calculated with the critical friction angle $\varphi_{\mathrm{c}}$. For a critical friction angle of $30^{\circ}$ the constants yield $c_{1}=0.1005, c_{2}=0.0782$ and $c_{3}=30.9483$.

In Fig. 2 results of 9 are compared with the relation found by Chu and Lo [3]

$$
\eta=\frac{q}{p^{\prime}}=M+\frac{M}{3} \tan \beta \quad \text { with } \quad M=\frac{6 \sin \varphi_{c}}{3-\sin \varphi_{c}}
$$

Here $\tan \beta=\frac{-\dot{\varepsilon}_{11}}{\dot{\varepsilon}_{\mathrm{v}}}$ is an alternative measure of dilatancy with

$$
\delta=\frac{\tan \beta}{\sqrt{1+\frac{(1+\tan \beta)^{2}}{2}}} .
$$

This relationship is experimentally well proofed.

\section{Proportional paths and fading memory}

Now we start from a stress state $\boldsymbol{T} \neq \mathbf{0}$ and apply the stretching $\boldsymbol{D}$. In order to asymptotically approach the corresponding proportional stress path $\boldsymbol{T}=\mu \boldsymbol{R}(\boldsymbol{D})$, the stress rate ${ }^{\circ}$ must be oriented towards a point of the proportional stress path with the direction $\boldsymbol{R}(\boldsymbol{D})$, i.e.

$$
\boldsymbol{T}+\lambda \stackrel{\circ}{\boldsymbol{T}}=\mu_{1} \boldsymbol{R}(\boldsymbol{D})
$$

where the positive constants $\mu, \mu_{1}$ and $\lambda$ need not be further specified at the moment. If we eliminate $\stackrel{\circ}{\boldsymbol{T}}$ we obtain an evolution equation for the stress: 


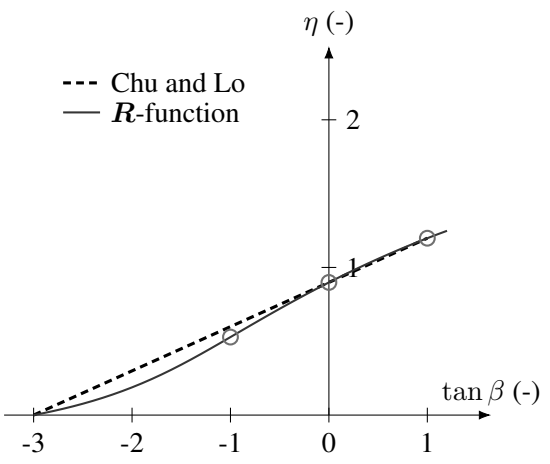

(a)

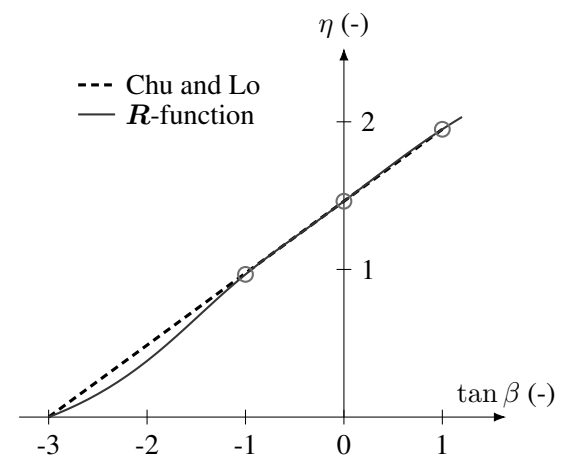

(b)

Fig. 2 Comparison of the stress-dilatancy relation from Chu and Lo with results obtained with the $\boldsymbol{R}$-function for $\varphi_{\mathrm{c}}=23^{\circ}$ (a) and $\varphi_{\mathrm{c}}=36^{\circ}$ (b).

$$
\stackrel{\circ}{\boldsymbol{T}}=v_{1} \boldsymbol{R}(\boldsymbol{D})+v_{2} \boldsymbol{T}
$$

with scalar quantities $v_{1}$ and $v_{2}$. Equation $(13)$ is already the general form of the barodetic constitutive relation. To comply with barotropy (i.e. dependence on $\sigma$ ), pyknotropy (i.e. dependence on $e$ ) and rate independence, $v_{1}$ and $v_{2}$ can be further specified, such that the barodetic constitutive equation obtains the following specific form:

$$
\stackrel{\circ}{\boldsymbol{T}}=h \cdot\left(f \boldsymbol{R}^{0}+g \boldsymbol{T}^{0}\right) \cdot\|\boldsymbol{D}\|
$$

where $\boldsymbol{R}$ is given by eq. 9. The quantities $f$ and $g$ will be specified below. $h$ is responsible for the stiffness and depends on $\|\boldsymbol{T}\|$. Subsequently it will be shown how all known concepts of soil mechanics can be cast in the frame given by equation (14).

\section{Limit states and peaks}

At limit states the stress rate vanishes:

$$
\stackrel{\circ}{\mathbf{T}}=\mathbf{0} \text {. }
$$

Limit states are manifested either as peak or residual (critical) limit states, where the stress-strain curves obtain a horizontal slope. In barodesy, $\dot{\boldsymbol{T}}=\mathbf{0}$ implies:

$$
f \boldsymbol{R}^{0}+g \boldsymbol{T}^{0}=\mathbf{0}
$$

This tensorial equation implies $3^{3}$

$$
\begin{aligned}
\boldsymbol{R}^{0} & =\boldsymbol{T}^{0} \\
f+g & =0
\end{aligned}
$$

\footnotetext{
${ }^{3}$ The other two possibilities, (i) $\boldsymbol{R}^{0}=-\boldsymbol{T}^{0}$ and $f-g=0$, as well as (ii) $f=0$ and $g=0$, can be excluded, because $\boldsymbol{R}$ always points to the compression octant, and $f$ and $g$ cannot vanish simultaneously, see equations 20 and 21
} 
Interestingly, equation (17) contains a flow rule, i.e. a stress-dilatancy relation for peak states. Critical limit states are obtained with $\delta=0$ and $e=e_{c}$, whereas peak limit states are obtained with $\delta>0$ and $e<e_{c}$.

\section{Incremental non-linearity}

Different stiffnesses at loading and unloading and, consequently, irreversible or hysteretic mechanical behaviour imply incremental non-linearity. Both, elastoplastic and hypoplastic relations exhibit incremental non-linearity. The elastoplastic approach consists in introducing (at least) two different stiffnesses, one for loading and one for unloading. A criterion has to be added to distinguish loading from unloading. In the frame of hypoplasticity and barodesy, a unique expression for the stress rate (or stiffness) is used, and the distinction between loading and unloading is accomplished by the non-linearity of this equation. In barodesy, the difference of stiffness at loading and unloading is modelled by the fact that the second term (i.e. $g \boldsymbol{T}^{0}$ ) in equation 14 is not changed if $\boldsymbol{D}$ is switched to $-\boldsymbol{D}$, whereas the first term (i.e. $f \boldsymbol{R}^{0}$ ) undergoes a change.

\section{Barodetic constitutive equations}

The main equation of barodesy is given by eq. 14 The scalar functions $h, f$ and $g$ obtain different representation for sand and clay.

\subsection{Sand}

For sand the scalar functions $h, f$ and $g$ of the barodetic constitutive equation are [13]:

$$
\begin{aligned}
h & =-\frac{c_{4}+c_{5}\|\boldsymbol{T}\|}{e-e_{\min }} \\
f & =\delta+c_{6} e_{c} \\
g & =-c_{6} e
\end{aligned}
$$

$e_{c}$ is a stress-dependent critical void ratio. Further details are given in [13]. The calibration must take into account the critical state line $e_{c}(p)$, the determination of which is still extremely difficult for sand.

\subsection{Clay}

In the open access article [19] a detailed description of the equations of barodesy for clay can be found. The scalar functions $h, f$ and $g$ in eq. (14) are: 


$$
\begin{aligned}
& h=c_{4}\|\boldsymbol{T}\| \\
& f=c_{6} \cdot \beta \cdot \delta-\frac{1}{2} \\
& g=\left(1-c_{6}\right) \cdot \beta \cdot \delta+\left(\frac{1+e}{1+e_{c}}\right)^{c_{5}}-\frac{1}{2} \\
& \text { with } \quad e_{c}=\exp \left(N-\lambda^{*} \ln \frac{2 p}{\sigma^{*}}\right)-1 \\
& \text { and } \quad \beta=-\frac{1}{c_{4} \Lambda}+\frac{1}{\sqrt{3}} 2^{c_{5} \lambda^{*}}-\frac{1}{\sqrt{3}} \\
& \text { with } \quad \Lambda=-\frac{\lambda^{*}-\kappa^{*}}{2 \sqrt{3}} \delta+\frac{\lambda^{*}+\kappa^{*}}{2}
\end{aligned}
$$

The constants $c_{i}$ can be calibrated on the basis of the critical state soil mechanics parameters $\varphi_{c}, N, \lambda^{*}$ and $\kappa^{*}$.

Isotropic loading and unloading of Weald clay is shown in Fig. 3 The calibration of the parameters $N, \lambda^{*}$ and $\kappa^{*}$ is illustrated in the $\ln (1+e)-\ln p^{\prime}$ plot Fig. 3 (b). The critical friction angle $\varphi_{c}^{\prime}$ is calibrated by a normally consolidated CU test. The four soil parameters $N, \lambda^{*}, \kappa^{*}$ and $\varphi_{c}^{\prime}$ are sufficient to calibrate barodesy for clay [19]18].

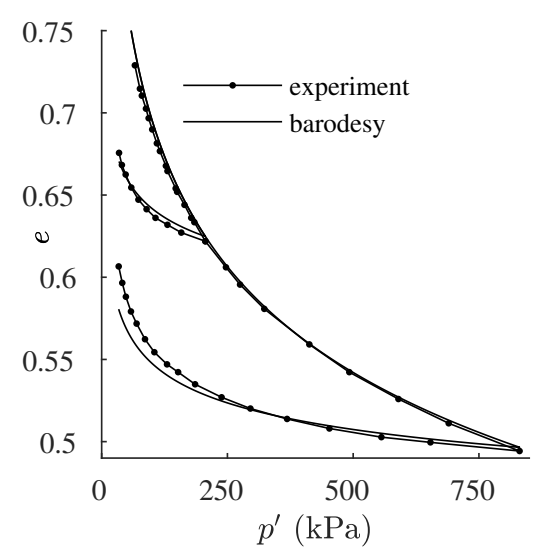

(a)

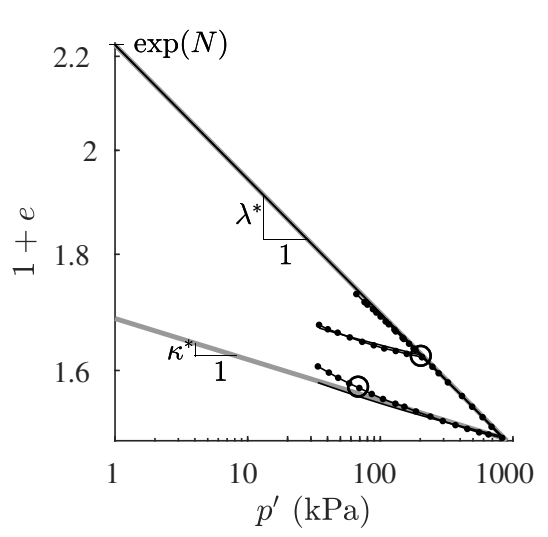

(b)

Fig. 3 Isotropic loading and unloading: Experimental results of Weald clay ([17], data from [8]) and numerical simulation with barodesy.

\section{Simulation of element tests}

In this section simulations of element tests with and without rotation of principal axes are shown. Element tests in general are idealizations and, in reality inhomogenities occur. Especially with shearing, localization takes place and the loss of homogeneity is unavoidable. 


\subsection{Rectilinear extensions}

\section{Proportional paths}

Applying various proportional strain paths starting at a non-vanishing stress state produces a fan of curved stress paths, all of which have to stay within the allowed range (fan) in the stress space. The corresponding graph (Figure 4 is a visualisation of the performance of a constitutive equation.

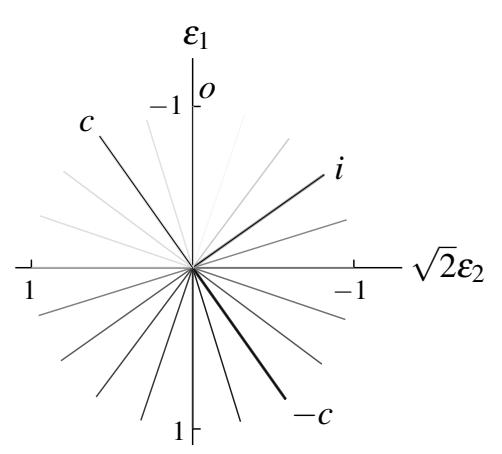

(a)

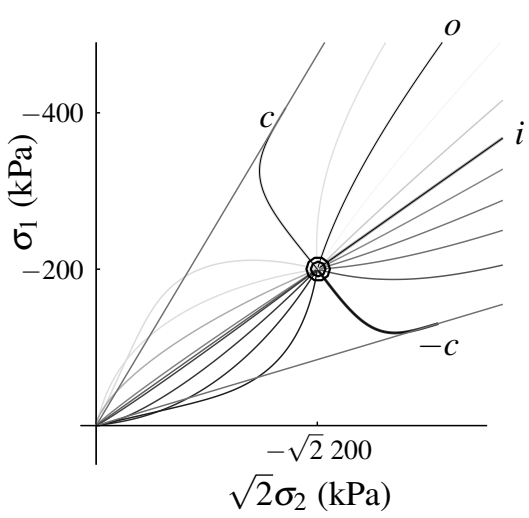

(b)

Fig. 4 Stress paths (b) corresponding to proportional strain paths (a) starting from a non-vanishing stress state. Note that stress paths asymptotically approach corresponding proportional stress paths. Weald clay $\left(\varphi_{c}=24^{\circ}\right)$ is simulated.

\section{Drained triaxial tests}

In Figures 5 and 6 simulations of drained triaxial tests with barodesy are shown. The highly overconsolidated/dense samples dilate to reach the critical state line. The normal and slightly overconsolidated/loose samples contract to reach the CSL. In Fig. 6 the highly overconsolidated samples (gray backed area) reach peak friction angles which are higher than the critical friction angle of $\varphi_{c}=24^{\circ}$.

\subsection{Rotation of principal stress and strain axes}

Fig. 7 presents a simulation from [19] of a simple shear test with a constant vertical stress of $\sigma_{y}=-100 \mathrm{kPa}$. The evolution of the shear stress $\tau_{x y}$ is plotted over the shear strain $\gamma$. The angle $\alpha_{\sigma}$ denotes the inclination of major principal stress to the horizontal direction $x$, and $\alpha_{D}$ is the inclination of major principal stretching. In Fig. 7 a Weald clay sample with initial stress $\boldsymbol{T}=$ 

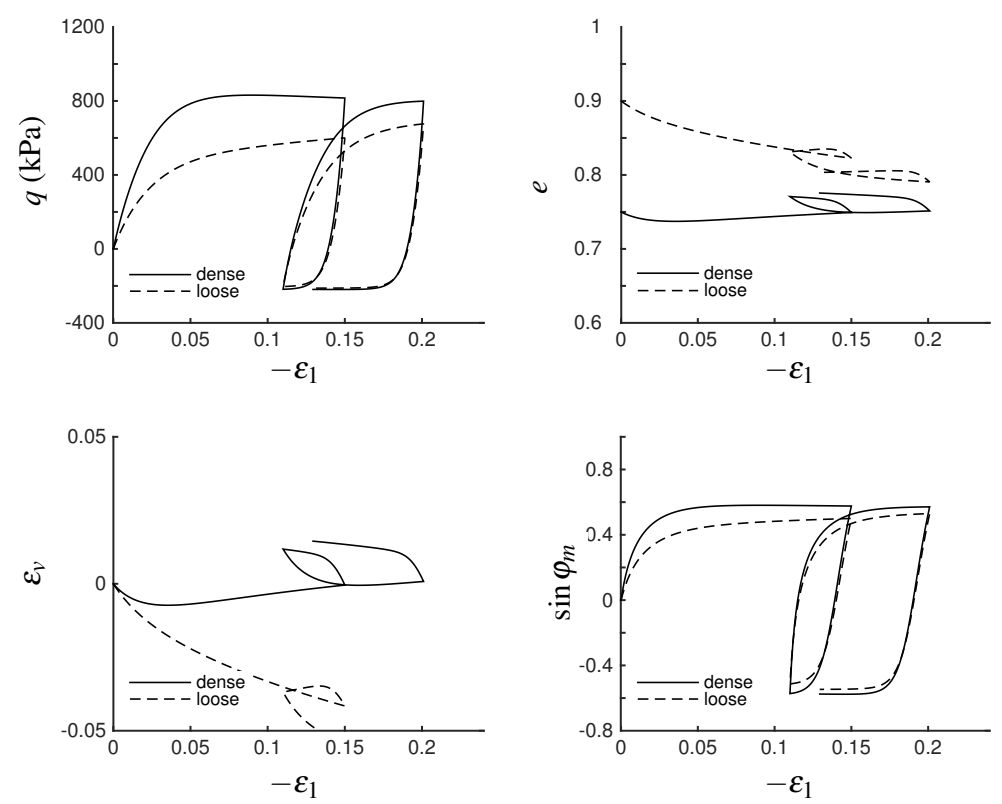

Fig. 5 Simulations with barodesy of drained triaxial tests

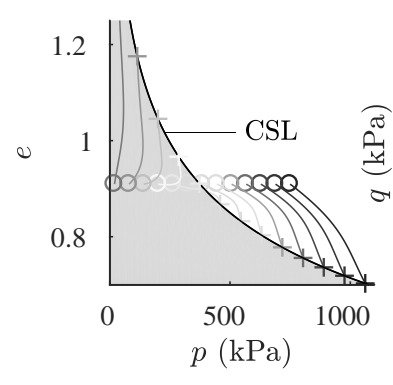

(a)

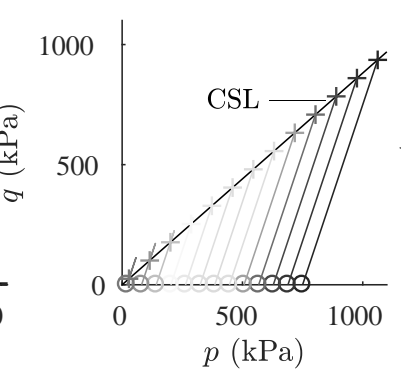

(b)

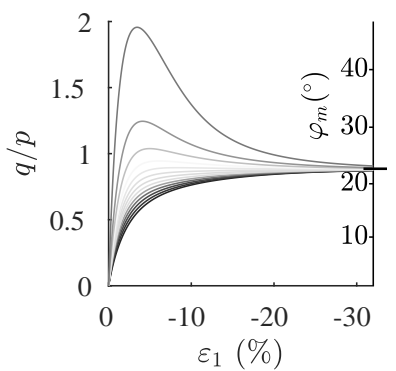

(c)

Fig. 6 Simulations with barodesy of drained triaxial tests of London clay $\left(\varphi_{c}=24^{\circ}\right)$. 
$\left(\begin{array}{ccc}T_{\text {ini }} & 0 & 0 \\ 0 & K_{0} \cdot T_{\text {ini }} & 0 \\ 0 & 0 & K_{0} \cdot T_{\text {ini }}\end{array}\right)$ is sheared. The major principal stress direction $\alpha_{\sigma}$ is $90^{\circ}$ at zero shear strain and decreases to $\approx 45^{\circ}$ with ongoing shear strain. The difference between the angles $\alpha_{D}$ and $\alpha_{\sigma}$, i.e., the angle of non-coaxiality $\alpha_{D}-\alpha_{\sigma}$ becomes very smal ${ }^{4}$, i.e., $\alpha_{\sigma} \approx \alpha_{D} \approx 45^{\circ}$ at the critical state. Similar results with hypoplasticity and an elasto-plastic model are shown in [23]. Experiments on sand according to [22] and DEM simulations [31,24] yield similar results, cf. [30].

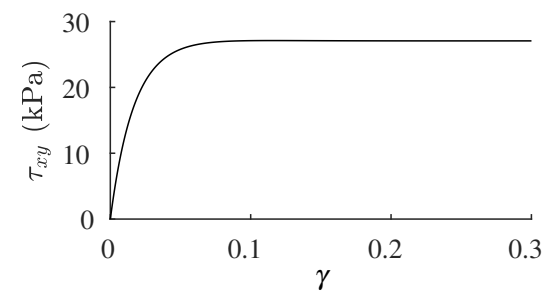

(a)

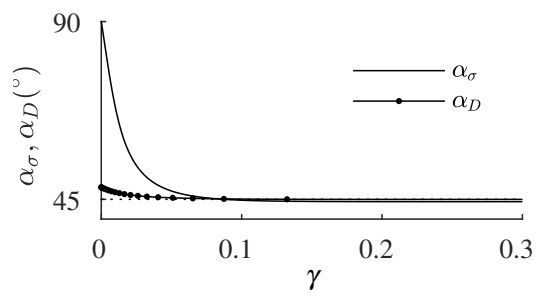

(b)

Fig. 7 Simple shear test with a constant vertical stress of $\sigma_{y}=-100 \mathrm{kPa}$, the initial radial stress is $\sigma_{x}=\left(1-\sin \varphi_{c}\right) \cdot \sigma_{y}=-59.33 \mathrm{kPa}$. In (b) directions of principal stress $\alpha_{\sigma}$ and principal stretching $\alpha_{D}$ are shown. Weald Clay with an initial void ratio $e_{\text {ini }}=0.68$ is simulated with barodesy. Figure from [19].

In Fig. 8 the evolution of the angle of non-coaxiality with ongoing shear strain is shown for different initial $K_{0}$ values. In Fig. 8] (a), DEM simulations from [31 24] show that the angle of non-coaxiality is small for $K_{0}=1$. For $K_{0}=2$, the angle of non-coaxiality decreases with ongoing shear strain to $\approx 0^{\circ}$; and for $K_{0}=0.5$ it increases to $\approx 0^{\circ}$. Zhang [31] states that non-coaxiality is significant before $10 \%$ shear strain. The predictions with barodesy in Fig. 8(b) are in good agreement with the DEM simulations in Fig. 8(a), which indicates that barodesy is applicable for general deformation, i.e., rotation of principal stress and strain axes.

\section{Conclusions}

In total, barodesy may be considered as attractive for its simplicity and elegance but has still potential for improvement, which should however preserve simplicity.

The present version of barodesy cannot cover all aspects of soil behavior. The memory of past loading is stored only in the actual stress $\boldsymbol{T}$ and the actual porosity $e$, and this is not sufficient to cover all aspects of re-loading, in particular the so-called aspects of 'small strain stiffness'. Though, it is interesting to note how many aspects of memory can be covered with $\boldsymbol{T}$ and $e$ only.

Acknowledgements The first author is supported by a research grant of the Austrian Science Fund (FWF): P 28934-N32

\footnotetext{
${ }^{4}$ At critical states $\alpha_{D}-\alpha_{\sigma} \approx 0.5^{\circ}$. Neglecting the Zaremba/Jaumann terms $-\boldsymbol{W} \boldsymbol{T}+\boldsymbol{W} \boldsymbol{T}$ yields $\dot{\boldsymbol{T}}=\dot{\boldsymbol{T}}$. It follows that $\alpha_{D}-\alpha_{\sigma}=0^{\circ}$ at failure.
} 


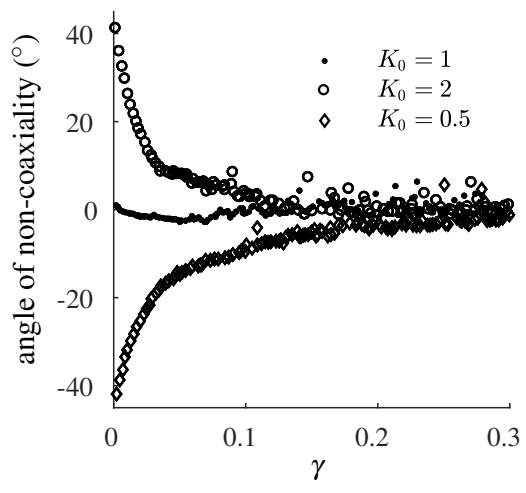

(a)

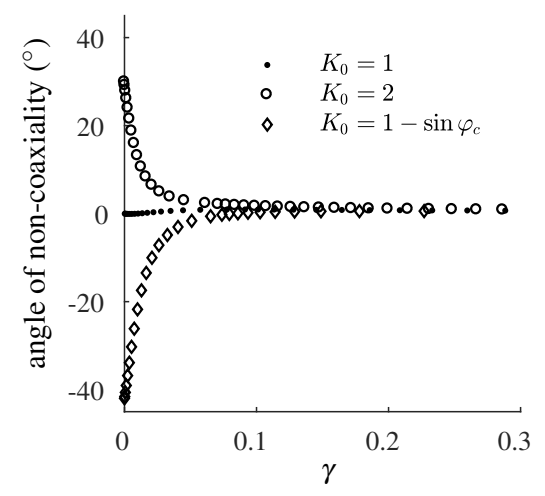

(b)

Fig. 8 Evolution of the angle of non-coaxiality in a simple shear test with different initial $K_{0}$ values: in (a), DEM simulations from [31 24] are shown, in (b), Weald Clay with $e_{\text {ini }}=0.68$ is simulated with barodesy. Figure from [19].

\section{References}

1. Bauer, E.: Calibration of a comprehensive hypoplastic model for granular materials. Soils and Foundations, 36(1), 13-26 (1996)

2. Bauer, E.: The critical state concept in hypopasticity. In: Comp. Meth. and Adv. in Geomechanics, Yuan (ed.) Balkema, 691-696 (1997)

3. Chu, J. and Lo, S.-C. R.: Asymptotic behaviour of a granular soil in strain path testing, Géotechnique, 44, 65-82 (1994)

4. Fellin, W.: Extension to barodesy to model void ratio and stress dependency of the K0 value. Acta Geotechnica 8(5), 561-565 (2013)

5. Fellin, W. and Ostermann A.: The critical state behaviour of barodesy compared with the Matsuoka-Nakai failure criterion. International Journal Numerical and Analytical Methods in Geomechanics, 37, 299-308 (2011)

6. Goldscheider, M.: Grenzbedingung und Fließregel von Sand, Mech. Res. Comm., 3, 463-468 (1976)

7. Gudehus, G.: A comprehensive constitutive equation for granular materials. Soils and Foundations, 36(1), 1-12 (1996)

8. Henkel, D.: The effect of overconsolidation on the behaviour of clays during shear, Géotechnique, 6, 139-150 (1956)

9. Herle, I. and Kolymbas, D.: Hypoplasticity for soils with low friction angles, Computers and Geotechnics 31, 365-373 (2004)

10. Kolymbas, D.: A rate-dependent constitutive equation for soils. Mech. Res. Comm. 4, 367372 (1977)

11. Kolymbas, D.: A generalised hypoelastic cosntitutive law. In: Proc. XI Int. Conf. Soil Mechanics and Foundation Engineering, Vol. 5, p. 2626, Balkelma, San Francisco (1985)

12. Kolymbas, D.: The misery of constitutive modelling. In: D. Kolymbas (ed.) "Constitutive Modelling of Granular Materials", Springer, 11-24 (2000)

13. Kolymbas, D.: Introduction to barodesy, Géotechnique, 65, 52-65 (2015)

14. Lade, P. V.: Effects of Consolidation Stress State on Normally Consolidated Clay, Rathmayer, H. (Ed.), Proceedings of NGM-2000: XIII Nordiska Geoteknikermötet, Helsinki, Finland, Building Information Ltd., (2000) 
15. Mašín, D.: Hypoplastic Cam-clay model, Géotechnique 62, 549-553 (2005)

16. Mašín, D.: Asymptotic behaviour of granular materials. Granular Matter, 14(6), 759-774 (2012)

17. Mašín, D.: Clay hypoplasticity with explicitly defined asymptotic states, Acta Geotechnica, Springer Berlin Heidelberg, 8, 481-496 (2013)

18. Medicus, G., Kolymbas, D. and Fellin, W.,: Proportional stress and strain paths in barodesy, International Journal for Numerical and Analytical Methods in Geomechanics, 40, 509-522 (2016)

19. Medicus, G. and Fellin, W.: An improved version of barodesy for clay, Acta Geotechnica, 12, 365-376 (2017)

20. Niemunis, A.: Extended hypoplastic models for soils. Heft 34, Schriftenreihe des Inst. f. Grundbau u. Bodenmechanik der Ruhr-Universitaet Bochum (2003)

21. Niemunis, A. and Herle, I.: Hypoplastic model for cohesionless soils with elastic strain range. Mechanics of Cohesive-Frictional Materials, 2(4), 279-299 (1997)

22. Roscoe, K., Bassett, R. and Cole, E.: Principal axes observed during simple shear of a sand, Proceedings of the 4th European Conference on Soil Mechanics and Geotechnical Engineering, 1, 231-237 (1967)

23. Schranz, F. and Fellin, W.: Stability of infinite slopes investigated with elastoplasticity and hypoplasticity, geotechnik, 39:3, 2190-6653 (2016)

24. Thornton, C. and Zhang, L.: A numerical examination of shear banding and simple shear non-coaxial flow rules Philosophical Magazine, 86, 3425-3452 (2006)

25. Topolnicki, M.: Observed stress-strain behaviour of remolded saturated clay and examination of two constitutive models. Veröffentlichungen des Instituts für Bodenmechanik und Felsmechanik der Universität Fridericiana in Karlsruhe (1987)

26. Truesdell, C. and Noll W.: The Non-Linear Field Theories of Mechanics. In: Encyclopedia of Physics, Vol. IIIc, Springer (1965)

27. von Wolffersdorff, P.-A.: A hypoplastic relation for granular materials with a predefined limit state surface. Mechanics of Cohesive-Frictional Materials, 1, 1, 251-271 (1996)

28. Wu, W.: Hypoplastizität als mathematisches Modell zum mechanischen Verhalten granularer Stoffe. Veröffentlichungen des Instituts für Bodenmechanik und Felsmechanik der Universität Fridericiana in Karlsruhe, Heft 129 (1992)

29. Wu, W., Bauer, E., Kolymbas, D.: Hypoplastic constitutive model with critical state for granular materials. Mechanics of Materials, 23, 45-69 (1996)

30. Yu, H.-S.: Plasticity and Geotechnics, Springer US (2006)

31. Zhang, L., The behaviour of granular material in pure shear, direct shear and simple shear Aston University, Aston University (2003) 